\title{
Galectins as Adaptors: Linking Glycosylation and Metabolism with Extracellular Cues
}

\author{
Michael Demetriou ${ }^{1}$; Ivan R. Nabi ${ }^{2}$; and James W. Dennis ${ }^{3,4}$ \\ ${ }^{1}$ Department of Neurology, University of California Irvine, 208 Sprague Hall, Mail Code 4032, Irvine, CA 92697, USA \\ ${ }^{2}$ Department of Cellular and Physiological Sciences, Life Sciences Institute, University of British Columbia, \\ 2350 Health Sciences Mall, Vancouver, BC, Canada V6T 1 Z3 \\ ${ }^{3}$ Lunenfeld Tanenbaum Research Institute, Mount Sinai Hospital, 600 University Avenue R988, Toronto, Ontario, Canada M5G 1X5 \\ ${ }^{4}$ Department of Molecular Genetics, \& Department of Laboratory Medicine and Pathology, Department of Medicine, University of Toronto \\ FAX: +1-416-586-8587, TEL: +1-416-586-8233, E-mail: dennis@lunenfeld.ca
}

(Received on March 8, 2018, accepted on March 27, 2018)

Key Words: galectin lattice, $N$-glycan remodeling, receptor dynamics, bioequivalence, metabolism

\begin{abstract}
Galectins interact with $N$-acetyllactosamine (LacNAc) epitopes in transmembrane glycoproteins at the cell surface in a multivalent manner forming a "lattice." The term "galectin lattice" was first used to describe the impact of galectin-3 on immune synapse formation, T cell activation and autoimmunity (Demetriou et al. (2001) Nature 409, 733). The galectin lattice displays rapid exchange of binding partners or stochastic-binding, thereby acting as an intermediary between free diffusion of glycoproteins and stable complexes in the membrane. This includes (i) slowing diffusion and loss of receptor and transporters to coated-pit endocytosis and/ or caveolin domains, (ii) slowing the integration of transmembrane phosphatases with signaling microdomains and (iii) promoting turnover (i.e., opposing stability) of cell-cell and focal adhesion complexes. The lattice model classifies galectins as adaptors of glycoprotein functions; regulating their localization, trafficking and thereby activity thresholds. The lattice model has been validated in immune regulation, cell adhesion and motility, and glucose homeostasis in mice. Here we review physical attributes of galectins and their $\mathrm{N}$-glycan ligands and apply logical inference, coupled with convergence of biochemical, cell biology and genetic evidence that provide a strong Bayesian probability for greater utility of the lattice model.
\end{abstract}

\section{A. Conserved but Ubiquitous}

Galectins (Gal) are a family of proteins characterized by a $\sim 130$ amino acid carbohydrate-recognition domain (CRD) that binds $\beta$-galactosides $(1,2)$, ligands found widely and at high density in the glycoprotein products of the secretory pathway $(3,4)$. The galectin gene family expanded early with the radiation of multicellular eukaryotes (5), along with the genes encoding Golgi $N$ acetylglucosaminyltransferases and $\beta$-galactosyltansferases, which generate LacNAc epitopes. Galectins are classified into the single CRD group (Gal-1, -2, -5, -7, -10, -11, -13), tandem-repeat (Gal-4, $-6,-8,-9$, and -12$)$. Gal-3 is unique, with a CRD and a $\sim 120$ amino acid N-terminal proline- and glycine-rich domain (6) which is intrinsically disordered (ID) and self-associates in a highly dynamic manner (7), with functionality similar to other low-complexity protein domains that mediate multivalent binding, and phase transitions forming liquid droplets in the cytoplasm (8-10). With specificity for the ubiquitous $\beta$-galactosides and self-association, galectins have the potential to crosslink transmembrane glycoproteins and form dynamic planar "lattices" with liquid droplet properties (Fig. 1) (reviewed in (11)). Gene duplication and evolutionary pressures have often resulted in divergent binding partners and activities within gene families. However, galectin CRD domains all appear to bind $\beta$-galactosides with varying affinities; represent- ing a highly conserved functionality in metazoans. There was also a 4-5 fold expansion of potential ligands in early metazoan evolution; genes encoding glycoproteins involved in morphogenesis (12). Galectin binding to $N$-glycans does not require contact with the underlying peptide sequence of the glycoprotein, but rather is dependent on the local concentrations of LacNAc ligands, discussed further below (13). This remarkable conservation of binding specificity suggests a functional imperative, but the ubiquitous distribution of the LacNAc sites, begs the question of how galectins contribute to specific cellular functions.

\section{B. Multivalency}

CRD domain affinities for the LacNAc disaccharide [Gal $\beta 1-3 / 4 \mathrm{GlcNAc}]$ are in the $100 \mathrm{uM}$ range (14) for Gal-1 and Gal-3, but binding avidity is greatly increased when density of LacNAc units are increased by multi-branched $N$-glycans, poly-LacNAc extension, and to large extent, by multiple $N$-glycan sites per glycoprotein, as suggested much earlier $(15,16)$. Galectin family members differ in affinities for LacNAc with $\alpha 2-3$ or $\alpha 2-6$ sialic acid and fucose substitutions (17) (See review by Iwaki and Hirabayashi in this series). The tissue-specific expression of enzymes catalysing branching and extension may shift galectin binding toward certain subsets of glycoproteins, and favour different 


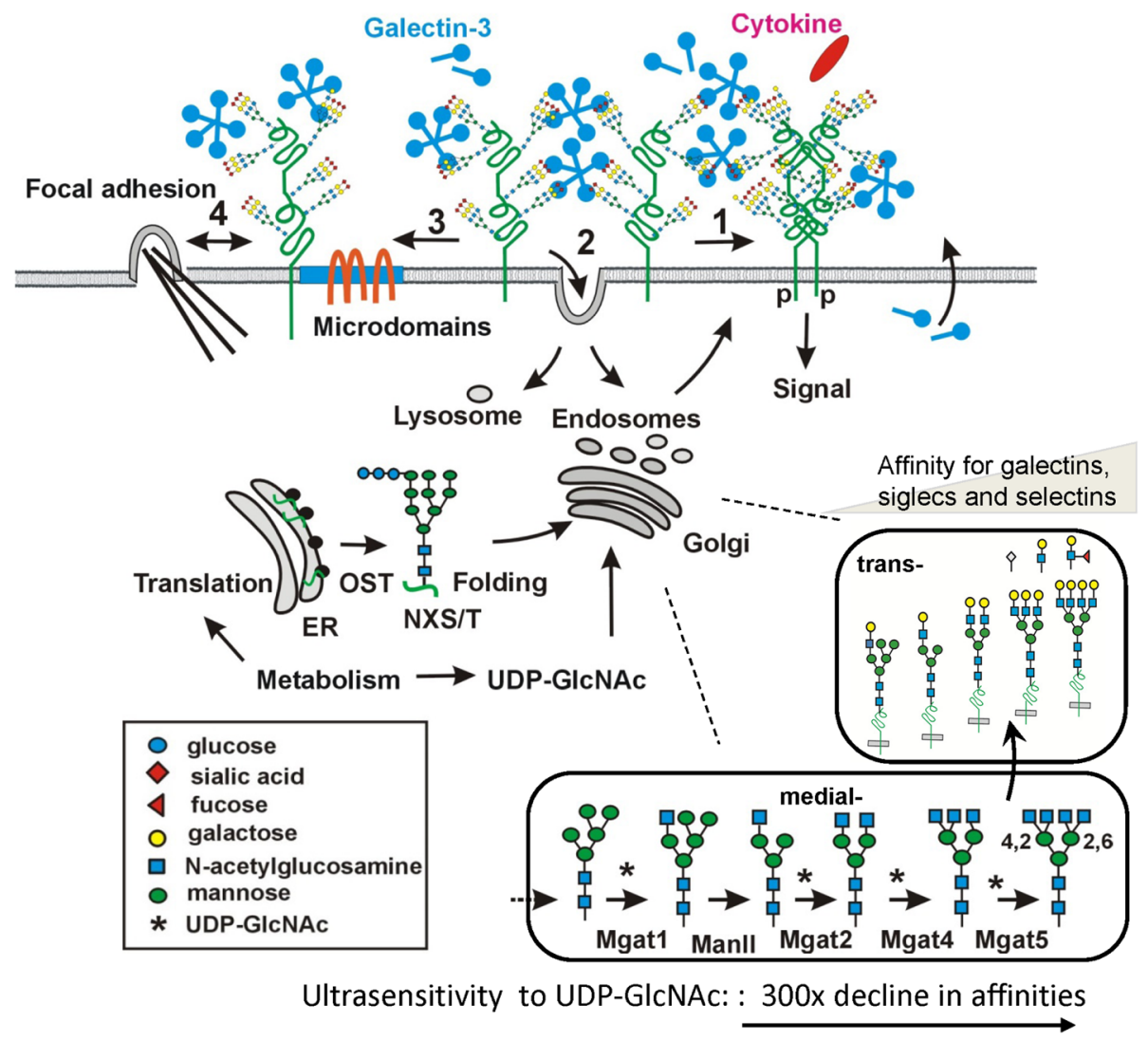

Fig. 1. $N$-glycan branching, galectin-lattice and regulation by metabolism. Galectins bind to $N$-acetyllactosamine (LacNAc) epitopes in Golgi remodeled $N$-glycans Affinities increase with ligand density driven by branching, extension and the number of NXS/T sites in glycoprotein sequences. Galectins gain access to the cell surface by a non-classical secretion mechanism. For simplicity, Gal-3 is shown as an NTD-NTD bound pentamer, which is only one of many possible microstates for self-association in the lattice. NMR and small-angle X-ray scattering analysis of Gal-3 self-association reveals stochastic binding, with many microstates states for complex formation (7). Gal-3 crosslinks transmembrane glycoproteins at the cell surface forming a lattice or liquid-droplet state that serves as an intermediate between stable complexes and free diffusion thereby; (1) inhibits clustering and precocious signaling by receptor tyrosine kinases (2), slowing loss of receptors to endocytosis (3), competing for receptor interaction with caveolae, Cav1 scaffolds and other microdomains, and (4) promotes turnover at cell-substratum and cell-cell adhesions. Galectin ligands are generated by glycosylation in the secretory pathway. Oligosaccharyltransferase (OST) modifies NXS/T $(X \neq P)$ sites in the lumen of the rough endoplasmic reticulum (ER). The medial Golgi $N$-glycan branching pathway is multistep ultrasensitivity to increasing UDP-GlcNAc; resulting in a switchlike increase in tri- and tetra-antennary $N$-glycans, thereby affinity for galectin binding.

members of the galectin family. However each glycoprotein is a population of glycoforms, with heterogeneity of glycan structures at each $N$-glycosylation site. The number of $N$-glycosylation sites $[\mathrm{NXS} / \mathrm{T}(\mathrm{X} \neq \mathrm{P})]$ is encoded in the sequence, but positions within the protein fold may influence the accessibility to Golgi remodeling enzymes $(18,19)$. Golgi $N$-acetylglucosaminyltransferases are also sensitive to metabolite supply to the hexosamine biosynthesis pathway (HBP) and flux of UDP-GlcNAc into the Golgi $(20,21)$.

\section{Background to the Lattice Model}

The term "galectin lattice" was first used to describe the Golgi $\mathrm{N}$-acetylglucosaminyltransferase V (Mgat5) mutation on immune synapse formation, $\mathrm{T}$ cell activation and autoimmunity in mice (22). A number of earlier observations lead to this milestone, and the lattice model of galectin function. In the early 1980s, JW Den- nis and RS Kerbel isolated and characterized lectin-resistant mutants from a metastatic lymphoma cell line, in an effort to assess the function of protein glycosylation in cancer growth and metastasis. Loss-of function mutations were selected in toxic concentrations of plant lectins (e.g., wheat germ agglutinin) (23). We found that mutation of the Golgi UDP-galactose transporter reduced solid tumor growth and lung colonization, and interestingly, tumor cell-host cell fusion events suppressed the recessive mutation and restored metastasis (24). Moreover, ectopic restoration of galactose to glycoproteins at the cell surface with UDP-galactose and recombinant $\beta 1-4$ galatosyltransferase also rescued organ colonization, suggesting that LacNAc plays a role in invasion and growth of blood born tumor cells (25). In these experiments, LacNAc was generated at the cell surface and not available for further modification in the Golgi. At this time, galectins were emerging as likely 
candidates for interactions with LacNAc, and particularly Gal-3 which was reported to be over-expressed in cancer (26-28). Oncogenic transformation of cultured fibroblasts increased the activity of tri- and tetra-antennary branching enzymes in the Golgi $\mathrm{N}$ glycan remodeling pathway (29-31). It also became clear that branched $\mathrm{N}$-glycans were the major binding sites on cultured mammalian cells. The Lec1 (Mgat1) mutation in $\mathrm{CHO}$ cells eliminates LacNAc in $N$-glycans, and reduced Gal-1, -3 and -8 binding to the cells by $\sim 90 \%$; a similar reduction was observed with Lec 8 mutant cells (UDP-Gal transport) which prevents galactose addition more broadly, on $\mathrm{N}$ - $\mathrm{O}$ - linked glycans and glycolipids (32). Mutation or shRNA knockdown of Golgi $N$-acetylglucosaminyltransferase I or V (encoded by Mgat1, Mgat5) inhibited cell migration as well as growth and metastasis in mice $(33,34)$. With the availability Mgat5-deficient mice and polyomavirus middle $\mathrm{T}$ transgene (MMTV-PyMT) cancer model (34), IR Nabi's group led studies revealing phenotypes in focal adhesion dynamics, cell motility and organization of extracellular matrix (35-37). We developed a computational model for regulation of growth factor receptor trafficking and signaling by $\mathrm{N}$-glycan remodeling and the galectin lattice $(20,38)$. In parallel, M. Demetriou's team studied the role of $\mathrm{N}$-glycan branching and metabolism in autoimmunity, and in the process validated the galectin lattice with cellular, biochemical and genetic approaches in mice and humans (39).

Mutation of Golgi branching $N$-acetylglucosaminyltransferases reduces LacNAc content in $N$-glycans affecting many glycoproteins at the same time, presumably resulting in complex phenotypes $(20,40,41)$. This is more than inference based on phenotypes of the knockout mice. We reported that tumor cells from Mgat5 $^{-1-}$ MMTV-PyMT mice (34) were less responsive to multiple growth factors including epidermal growth factor (EGF), fibroblast groth factor (FGF), platelet derived growth factor (PDGF) and transforming growth factor (TGF- $\beta$ ) (38), as well as $\beta 1$ integrin-dependent signaling $(36,37,42)$. We confirmed that $N$-glycans on EGF and TGF- $\beta$ receptors were ligands for Gal-3, and binding was weaker in $\mathrm{Mgat} 5^{-/-}$cells resulting in loss at the receptors from the surface and accumulation in the endosomes. Cytokine signaling could be rescued by (i) restoring Mgat5 expression, (ii) inhibition of constitutive endocytosis (38), (iii) GlcNAc supplementation which increases UDP-GlcNAc and activity of enzymes earlier in the branching pathway (20), (iv) adaptive changes in the cells including loss of caveolin-1 expression, which is a negative regulator of EGFR sensitivity $(20,43)$, and (v) altered oxidative phosphorylation allowing constitutive signaling by reactive oxygen species (ROS)-dependent inhibition of phosphatase-and-tensinhomolog (PTEN) and other phosphatases (44).

With these observations, our goal became a systems-level model of cell surface glycoproteins regulated by galectins and $\mathrm{N}$ glycans (20). This is contrary to most of the galectin literature to date, where the identification of galectin-specific interactions is the goal. This reductionist approach has diminishing returns in the current research environment where multivalent interactions and phase transition systems are being observed widely (11).

\section{Lattice Model}

Most glycosylated NXS/T $(\mathrm{X} \neq \mathrm{P})$ sites are occupied with a biosynthetically-related distribution of $\mathrm{N}$-glycan structures. The $\mathrm{N}$ glycans can be ordered by LacNAc units and affinities for specific galectins (20), based on available data (14) (See review by Iwaki and Hirabayashi in this series). $N$-glycosylation begins in the ER where the glycan portion of $\mathrm{Glc}_{3} \mathrm{Man}_{9} \mathrm{GlcNAc}_{2}$-pp-dolichol is cotranslationally transferred to Asn at $\mathrm{NXS} / \mathrm{T}(\mathrm{X} \neq \mathrm{P})$ sites in the nascent protein. After the removal of glucose and mannose residues, the $N$-glycans are modified in the medial Golgi by $N$-acetylglucosaminyltransferases (encoded by MGAT1, MGAT2, MGAT4a,b,c, MGAT5). The affinity for UDP-GlcNAc declines 300 fold, from Mgat1 to Mgat5; thus the pathway to tri- and tetra- antennary products is ultrasensitive to UDP-GlcNAc (Fig. 1) (20). The GlcNAc antennae are substrates for $\beta 1,4$ galactosyltransferases, which generates LacNAc antennae and then extended in the trans Golgi to varying degrees as noted above (14). Heterogeneity at each site is due in part to competition between enzymes for acceptor substrates (45), nucleotide-sugar levels (20), as well as cooperative association between enzymes (46), and transit rates of glycoprotein substrates in the Golgi (47). Glycoproteins with $n$ posttranslational modification (PTM) sites have an exponential number $\left(\mathrm{n}^{\mathbf{X}}\right)$ of possible isoforms (48), where developmental and environmental conditions determine the cumulative output $\Sigma \mathrm{p}_{\mathrm{i} . \ldots \mathrm{x}}$ of the Golgi pathway (49); generating too many glycoforms to evaluate individually (50). However, the glycoform distribution can be estimated based on a knowledge of Golgi pathway activity and affinities of glycans for galectins (14), and used to compute the relative changes in glycoforms, their interaction with galectins.

We constructed an Ordinary Differential Equation (ODE) model with estimates of intracellular enzyme concentrations and kinetic parameters from the literature as outlines (20). We perturbed parameters in silico and experimentally, changing UDPGlcNAc supply, branching enzyme activities, trafficking of substrate glycoproteins (Fig. 2A). The results confirmed that ultrasensitivity (Hill coefficient, $\mathrm{Hn}$ ) was distributed over multiple reactions; requiring the $\sim 300$ fold decline in affinity for UDP-GlcNAc from Mgat1 to Mgat5, and is a robust property of the pathway. Importantly, the model predicts that a gain of Mgat1 activity should reduce the availability of UDP-GlcNAc to Mgat4 and Mgat5, 


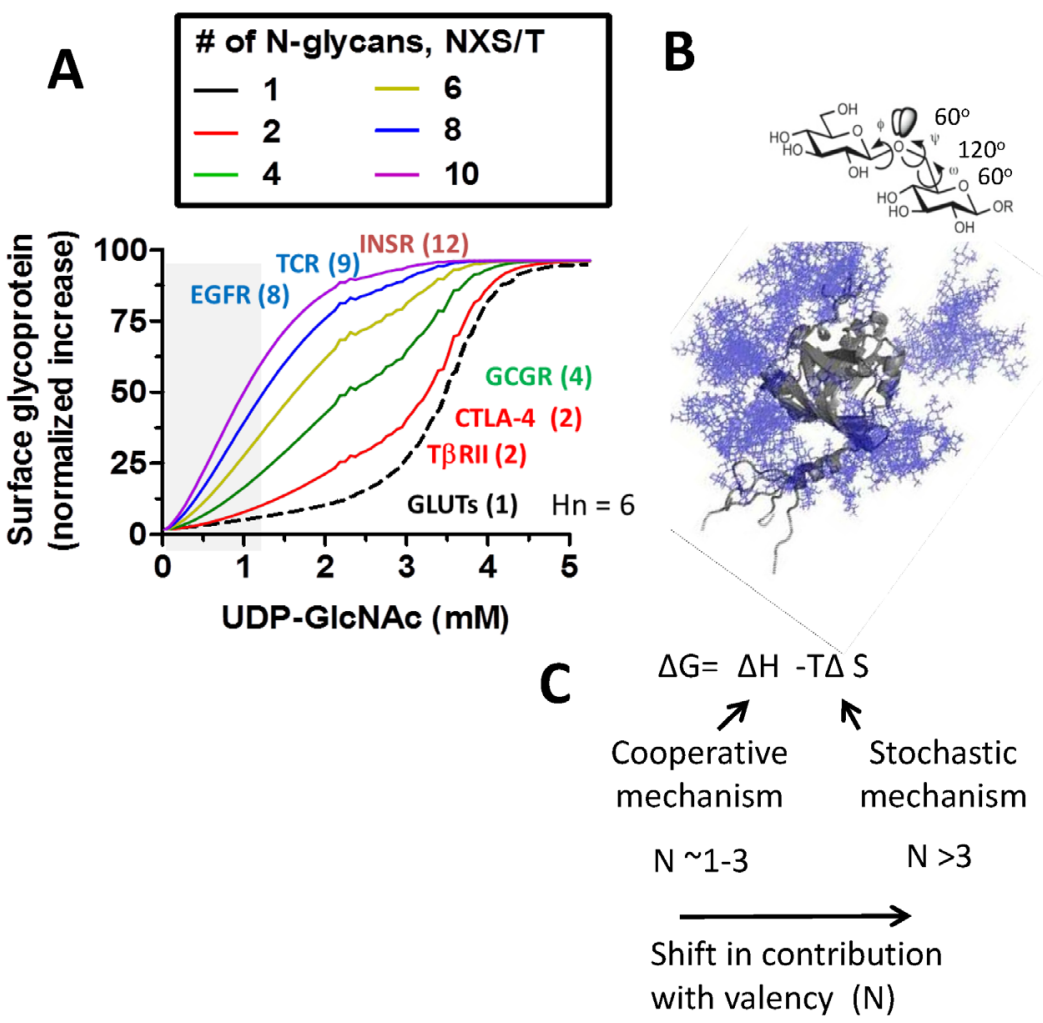

Fig. 2. The functional state of the galectin lattice is a dynamic ensemble. (A) Simulation of HBP-dependent regulation of surface levels of receptors by the galectin lattice in tumor cells using an ordinary differential equation model. Endocytosis was modeled as the major lattice-opposing force. The degree of ultrasensitivity (Hill coefficient) to increasing UDP-GlcNAc/ $N$-glycan branching is inversely proportional to NXS/T $N$-glycan site multiplicity (modified from Lau. et al (20)). Ultrasensitivity of the $N$-glycan branching pathway to UDP-GlcNAc allows sequential enrichment of receptor in the lattice based on NXS/T multiplicity, thereby an order of opposing signals, for example growth then arrest. (B) $N$-glycan and glycoprotein microstates: Small-angle X-ray scattering (SAXS) structural data applied to all-atom modeling of five $N$-glycan chains on $\alpha 1$-acid glycoprotein (183 aa), an overlay of the top five models in the ensemble (modified from Guttman et al. (63)). (top) Rotational angles in the three bond of 1-6 linked disaccharide. (C) The flexibility of glycan polymers, multivalency and galectin affinities that are tuned for dynamic "jumping" between sites (i.e., stochastic binding - with microsecond $\mathrm{T}_{1 / 2}$ ) allows for a large entropic contribution to the lattice structure (modified from (93)).

thus depleting the higher-affinity galectin-3 ligands (tri- and tetraantennary), while increasing lower-affinity bi-antennary. This was confirmed in non-transformed cells, while increased expression of Mgat4 and/or Mgat5 in cancer cells suppressed the ultrasensitive response to UDP-GlcNAc (51). Endocytosis and Gal-3 binding were assumed to be non-limiting quantities and modeled as linear processes (20). Therefore, the ODE model could be expanded to include variables accounting for expression and activity of specific galectin family members.

\section{E. Bio-Equivalence}

A further blow to those seeking specificity is the remarkable amounts of bioequivalence of glycans generated within and between biosynthesis pathways $(13,20,40,52)$. The first demonstration of bioequivalence was the rescue of surface receptors in Mgat5 deficient cells by increasing LacNAc content in $N$-glycans through exogenous supply of GlcNAc, which is taken up by fluid phase endocytosis and salvaged into HBP to UDP-GlcNAc (20).
Mgat5 has no gene homologues, but compensating amounts of $\mathrm{N}$-acetyllactosamine branches are produced by supplementing $\mathrm{Mgat}^{-/-}$cells cultures with GlcNAc, which increases UDPGlcNAc and the activities of Mgat4a,b,c and poly $N$-acetyllactosamine extension enzyme, and B3GNT-1,-3 family (20, 53). The Golgi branching enzymes function together as an ordered linear pathway, each transferring GlcNAc from UDP-GlcNAc to specific positions on the $N$-glycans. The degree of ultrasensitivity is inversely proportional to site multiplicity. For example, the glucose transporters GLUT4 has one $N$-glycan and titration of GlcNAc in cell culture increased cell surface expression with a switch-like response matching the global increase in tetra-antennary $N$-glycans (i.e., Hn 10) (Fig. 2A) (20). Insulin release by pancreatic $\beta$-cells is also dependent on $\mathrm{N}$-glycan branching on GLUT2 transporter and the galectin lattice $(54,55)$.

Bio-equivalence was also recently demonstrated between hybrid and complex $N$-glycans based on their LacNAc content (53). Deficiency of medial Golgi enzyme Mgat2 in T cells trig- 

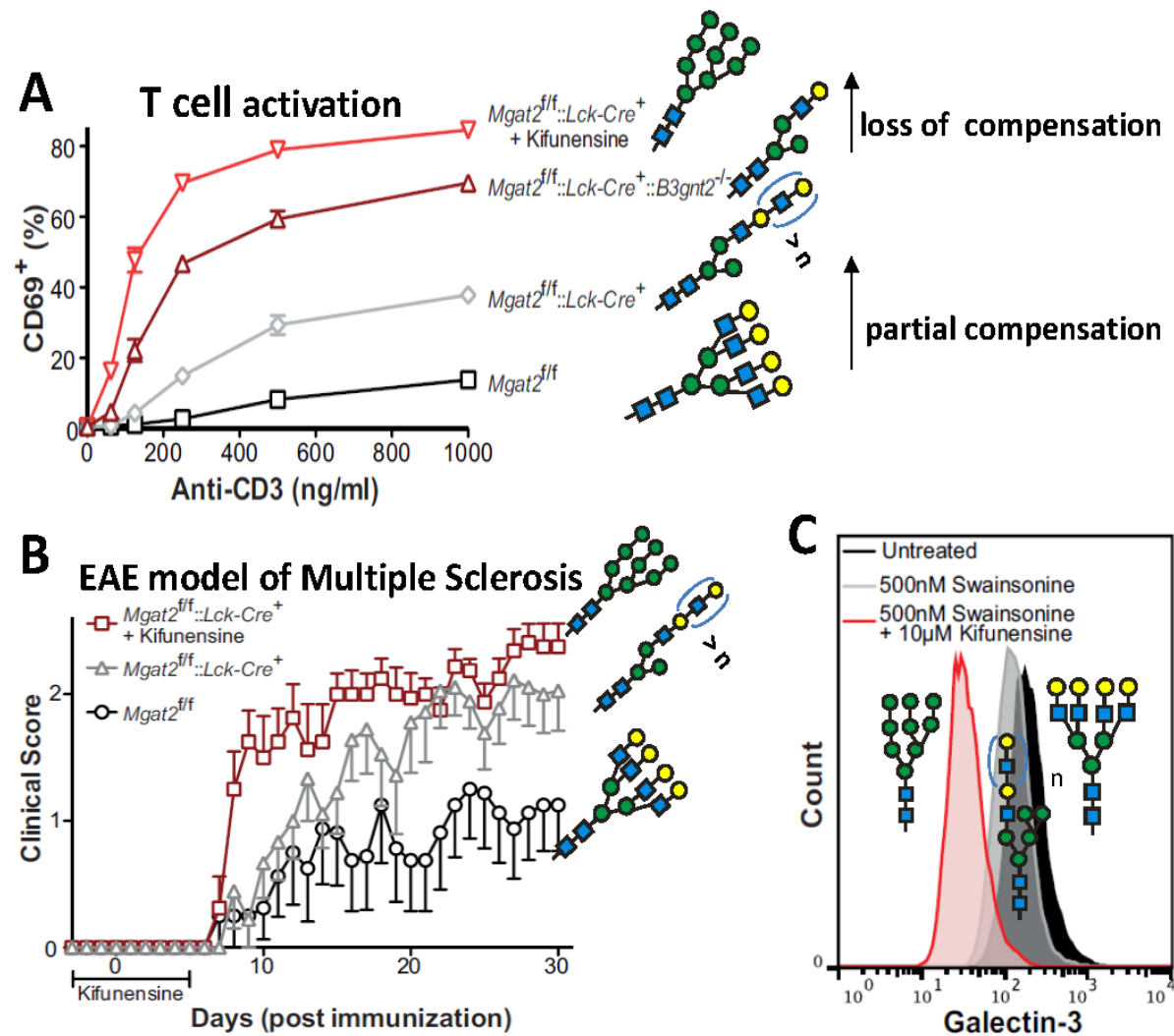

Fig. 3. Bioequivalence of hybrid and complex $N$-glycans in immune cells by rearranging LacNAc units. (A) Mouse $\mathrm{T}$ cells of the indicated genotypes and varying placement and number of LacNAc units in $\mathrm{N}$-glycans were activated for $24 \mathrm{~h}$ with plate bound anti-CD3 and analyzed for CD69 expression by flow cytometry, gating on $\mathrm{CD}^{+}$cells. Where indicated, mice were pre-treated for 3 days with $0.2 \mathrm{mg} / \mathrm{mL} \mathrm{kifunensine} \mathrm{in} \mathrm{the}$ drinking water followed by $10 \mu \mathrm{M}$ kifunensine during culture, thereby preventing placement of LacNAc in $N$-glycans. (B) Autoimmune encephalomyelitis (EAE) was induced in age-matched female C57BL/6 mice of the indicated genotypes resulting in poly-LacNAc extended hybrid or branched complex $N$-glycans were treated with or without kifunensine in the drinking water at $0.2 \mathrm{mg} / \mathrm{mL}$ from day -3 to 5 , with day 0 indicating the time of immunization ( $n=9$ per group) and assessed daily for clinical signs of EAE. (C) Jurkat T cells were treated as indicated for $72 \mathrm{~h}$ in culture and analyzed for galectin-3 binding by flow cytometry (modified from (53)). Mannosidase II/IIx inhibition by swainsonine induces hybrid $N$-glycans with poly-LacNAc extension, which is blocked by mannosidase I inhibition with kifunensine.

gered poly-LacNAc extension of the single GlcNAc branch in hybrid $N$-glycans by the trans- Golgi $\beta 3$ GNT enzymes, resulting in bio-equivalence to bi- and tri-antennary structures. Remarkably, the data suggested poly-LacNAc extension is triggered by unused UDP-GlcNAc fluxing from the medial to trans Golgi as a result of hypo-active medial Golgi branching enzymes, thereby enhancing $\beta 3 \mathrm{GNT}$ enzyme activity. In this manner, evolutionarydriven placement of branching enzymes in the medial Golgi versus $\beta 3$ GNT extension enzymes in trans-Golgi provides a fail-safe mechanism to maintain LacNAc content within $N$-glycans. Bioequivalence plays a critical role in regulating the sensitivity of $\mathrm{T}$ cells to autoantigens and prevention of autoimmunity in mice (Fig. $3 \mathrm{~A}-\mathrm{C})$.

Recently, signal peptide peptidase-like 3 (SPPL3), an intramembrane-cleaving aspartyl protease of the GxGD type, was shown to release the ectodomains of type II membrane proteins, notably Golgi enzymes including $N$-acetylglucosaminyltransfer- ases (MGAT5, MGAT2, B3GNT-1,-3) $\beta$-1,4 galactosyltransferase 1 (B4GALT1) and $\alpha$-mannosidase 2A1 (MAN2A1) $(56,57)$. Reduced expression of SPPL3 results in a hyper-glycosylation phenotype, whereas elevated SPPL3 expression causes hypoglycosylation, and may be associated with conditions of metabolic stress. Interestingly, SPPL3 targets multiple Golgi enzymes, consistent with a requirement to regulate $N$-glycan redundancies and bioequivalence at a cellular level.

\section{F. Lattice Dynamics}

The first demonstration of galectin lattice dynamics was the use of exogenous soluble lactosamine to disrupt the interaction of endogenous Gal-3 with the T cell-receptor (TCR) (22). Soluble lactosamine phenocopied the Mgat5 deficiency, enhances both TCR clustering and signaling at the immune synapse (58). This demonstrated for the first time that $N$-glycan branching regulated the adaptor function of galectins, rather than the prevailing dogma 
that $N$-glycans alter function via changes in protein structure or ligand binding affinity. Galectins were subsequently shown to preferentially bind glycoproteins on surfaces rather than in solution, the former driven by a threshold concentration of multivalent ligands (59). Tandem-repeat galectins readily cross-link glycoproteins, single CRD galectins can dimerize as exemplified by Gal-1 (59). For Gal-3, CRD binding to multivalent glycoproteins promotes the intrinsically-disordered N-terminal domain (NTD) to self-associate via amino acid residues $~ 20-100$, accompanied by NTD binding to a surface on the CRD which is not involved in carbohydrate recognition $(7,60,61)$. Gal-3 mixed with a bivalent $\mathrm{N}$-glycan ligand in vitro at optimal concentrations, undergoes phase transition to a liquid-droplet state, which is NTD dependent and can be inhibited by monovalent $\beta$-galactosides (62). Factors that contribute to liquid-droplet state of the lattice include multivalency, irregular geometry and entropic contribution to binding (Fig. 2C). In live cells, the lattice is planar liquid-droplets, held in proximity to the membrane by transmembrane glycoproteins. The diversity of glycoproteins contribute to a disordered geometry and heterogeneity of the galectin lattice (11). Furthermore, $N$-glycans generally extend away from the protein surface and display the entropy of a flexible polymer (63) (Fig. 2B, C). The Gal-3 CRD also displays conformational entropy which contributes favorably to the multivalent stochastic binding of ligand $(64,65)$. Gal-3 and Gal9 cross-linking of transmembrane glycoproteins can slow lateral diffusion of freely diffusing glycoproteins as measured by fluorescence recovery after photobleaching (FRAP) (43). Manipulating galectin levels, $\mathrm{N}$-glycan branching activities or endocytosis alters

\section{Gal-9 lattice slows glucagon receptor dynamics in hepatocytes}

A

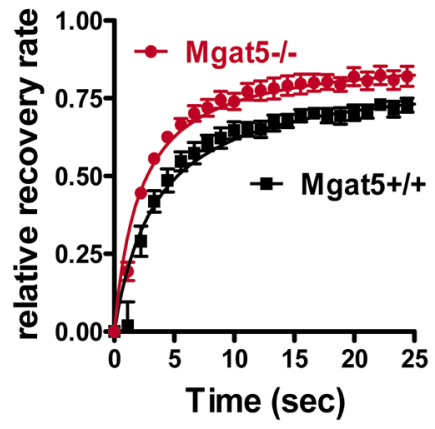

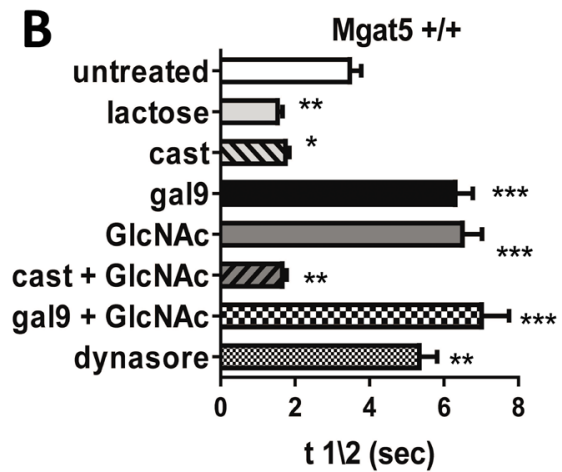

$\mathrm{t} 112$ (sec)

\section{Gal-3 lattice promotes focal adhesion dynamics}

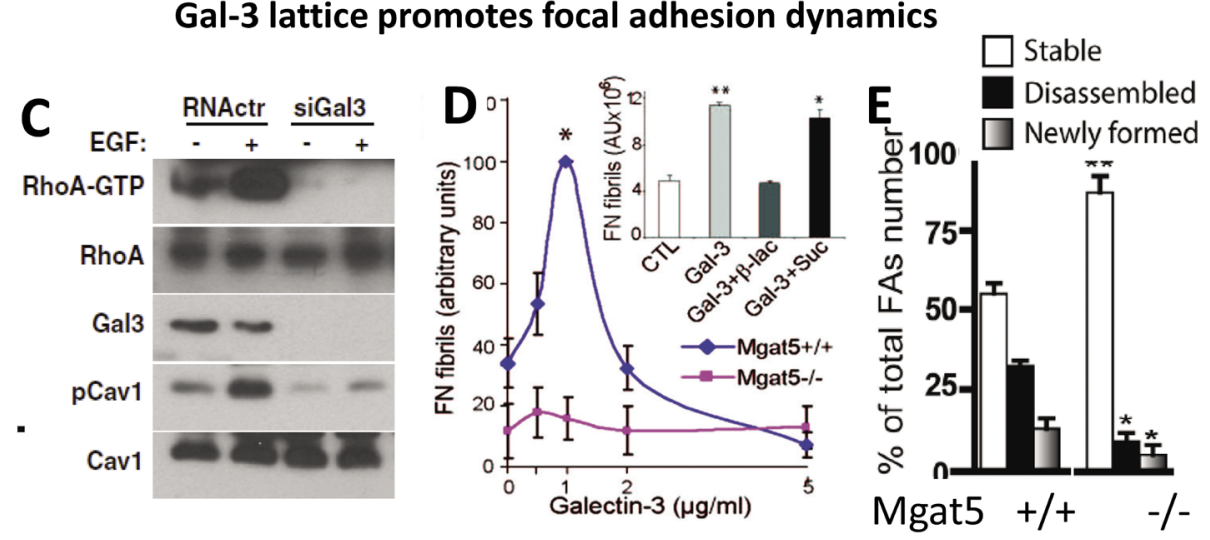

Fig. 4. Galectin lattice regulates the dynamics of hepatic glucagon receptor and focal adhesions. (A) Time course of fluorescence recovery after photobleaching (FRAP) of GFP-tagged glucagon receptor in primary mouse hepatocytes $(n=5)$. (B) Half-life $\left(\mathrm{T}_{1 / 2}\right)$ for recovery of GFP-tagged glucagon receptor in primary hepatocytes pre-treated as indicated with either $30 \mathrm{mM}$ lactose, $100 \mu \mathrm{M}$ castanospermine, and $20 \mathrm{mM}$ GlcNAc; $(n=5$ independent experiments, 6-10 cell bleached/experiment) (modified from (40)). Lack of interaction with the galectin lattice in Mgat5 ${ }^{-/-}$hepatocytes increases receptor mobility. (C) MDA-MB-231 cells were transfected with control (ctr) or Gal3-targeted (Gal3) siRNA for $48 \mathrm{~h}$ followed by treatment with EGF for $5 \mathrm{~min}$; lysates were subjected to Rhotekin-RBD pull down for activated RhoA (RhoA-GTP) relative to the total amount of RhoA, and western blotted for Gal-3, Cav1 and Y14-phosphorylated pCav1 levels (modified from (66)). (D) Fibronectin polymerization by Mgat5 ${ }^{+/+}$and

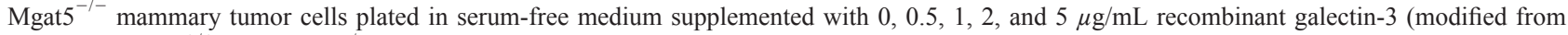
(36)). (E) Mgat5 $5^{+/+}$and Mgat5 $5^{-/-}$mammary tumor cells were transfected with FAK-GFP and imaged every 30 s for 30 min. Overlay of FAK-GFP images at time 0 (red) and $30 \mathrm{~min}$ (green) allowed quantification of disassembled (red), stable (yellow), and newly formed (green) FAs (modified from (37)). The galectin lattice therefore promotes focal adhesion signalling, turnover and matrix re-modeling. 
FRAP half-life of glucagon receptor at the cell surface in a manner consistent with the lattice model (40) (Fig. 4A, B). Exogenous galectin promotes the turnover of $\beta 1$-integrin-dependent focal adhesion signaling motility as well as fibronectin polymerization (Fig. 4C-E). These responses to increasing galectin concentrations were non-monotonic. The titration of recombinant Gal-3 stimulates cell migration and fibronectin remodeling (36) at an optimal concentration, above which Gal-3 inhibits $(36,66,67)$. The biphasic response to Gal-3 and Gal- $8(68,69)$ is analogous to precipitation responses to divalent antibody, and highlighting the importance of galectins as multivalent adaptors. Lattice dynamics have also been measured by fluorescence resonance energy transfer (FRET) (70), and single-particle tracking (71).

\section{G. Emergent Properties of the Galectin Lattice}

How does the galectin lattice provide a selective advantage to organisms? In proliferating and motile cells there is often high rates of membrane turnover, and the galectin lattice opposes receptor internalization (38), but does so unequally in proportion to $\mathrm{NXS} / \mathrm{T}(\mathrm{X} \neq \mathrm{P})$, an evolved feature of each receptor (20). For example, epidermal growth factor receptor (EGFR) with eight occupied sites, stimulates metabolism including UDP-GlcNAc biosynthesis, and this ultimately recruits TGF- $\beta$ receptors with two $N$-glycans into the lattice allowing increased autocrine TGF- $\beta / \operatorname{Smad} 2 / 3$ signaling and suppression of cell proliferation (20). The ODE model and experimental validation agreed well in this and other examples where differences in $\mathrm{NXS} / \mathrm{T}(\mathrm{X} \neq \mathrm{P})$ number between receptors that signal in opposite directions creates a UDP-GlcNAc-dependent delay in their recruitment to the lattice. Receptors with low site number are recruited at a later stage in the titration of UDP-GlcNAc and branching. Examples include T cell activation followed by arrest (39) and hepatic glycolysis balanced against gluconeogenesis $(40,72)$. Thus, $N$-glycan biosynthesis and biophysical properties of the galectin lattice provide a selective advantage to animals in the form of tunable and ultrasensitive stimulus-response relationships.

The galectin-lattice model has been validated in several biological settings. In T cells, the lattice suppresses $\mathrm{T}$ cell receptor signalling to control antigen-induced activation and positive selection in the thymus to promote self-tolerance and prevent autoimmunity $(22,73,74)$. In humans, single nucleotide polymorphisms (SNPs) in MGAT1, MGAT5 and CTLA4 genes are associated with Multiple Sclerosis $(39,75)$. Gain-of-activity SNPs in the coding sequence of Mgat1 increase expression and when coupled with $\sim 300$ fold higher affinity of Mgat1 for UDP-GlcNAc relative to Mgat5; this reduces UDP-GlcNAc availability to Mgat5, which suppresses LacNAc content and galectin-dependent regulation of $\mathrm{T}$ cells. The Mgat1 SNPs interact with a SNP in CTLA-4 that reduces $N$-glycan site occupancy, for a combined increase in Multiple Sclerosis risk. Together, they decrease lattice recruitment of CTLA-4 to the cell surface, thereby delaying its action as a negative regulator of $\mathrm{T}$ cell proliferation (20). CTLA-4 levels are rescued by GlcNAc supplementation in tissue culture, and in vivo oral GlcNAc also suppresses experimental autoimmune disease in mice and rescues signaling consistent with the model $(76,77)$. Oral GlcNAc in MS patients is currently in clinical trials led by Michael Demetriou. These observations and a genome-wide evolutionary analysis of $\mathrm{NXS} / \mathrm{T}(\mathrm{X} \neq \mathrm{P})$ sites is consistent with selective pressures on subsets of glycoproteins in opposing directions (i.e., high or low site number) $(78,79)$. This is an emergent property associated with the evolution of Golgi $\mathrm{N}$-glycan processing and galectins as adaptors of receptors regulating opposing pathways.

\section{H. Metabolic Regulation of the Lattice}

Ultrasensitivity of the branching pathway and LacNAc content within $N$-glycans depends on the production of UDP-GlcNAc, which can be taken advantage of therapeutically through GlcNAc supplementation and salvage into the HBP. However, de novo synthesis of UDP-GlcNAc from glucose provides another critical mechanism regulating lattice strength. The HBP directly competes with glycolysis for available glucose (Fig. 5). The most significant metabolic change in cells is a switch from complete oxidation of glucose that produces 36-38 ATP (oxidative phosphorylation) to fermentation of glucose to lactate and generation of only 2 ATP. This switch is induced when oxygen is limiting (anaerobic glycolysis) but is paradoxically also induced in rapidly dividing cells despite sufficient oxygen (aerobic glycolysis), a phenomenon first reported in cancer cells by Otto Warburg over 90 years ago. Aerobic glycolysis is often accompanied by glutaminolysis, where glutamine is converted to $\alpha$-ketoglutarate for flux into the TCA cycle. Glutamine is required for de novo synthesis of UDP-GlcNAc by the HBP, acting as an amine donor in the rate limiting conversion of fructose-6-phosphate to glucosamine-6-phosphate. In blasting $\mathrm{T}$ cells, aerobic glycolysis and glutaminolysis cooperatively reduce de novo UDP-GlcNAc production by limiting flux of glucose and glutamine into the HPB. This lowers $N$-glycan branching and lattice strength to drive $T$ cell growth and pro-inflammatory $\mathrm{T}_{\mathrm{H}} 17$ over anti-inflammatory $\mathrm{T}$ regulatory (iTreg) differentiation by lowering IL-2R $\alpha$ (CD25) surface levels $(52,77)$ (Fig. 5). In this manner, aerobic glycolysis and glutaminolysis serve as critical negative regulators of lattice strength, receptor content and cell fate.

\section{Galectins as Adaptors of Glycan Mimics in the $\mathbf{C y}$ - toplasm}

The primordial function of galectins may have been the ca- 


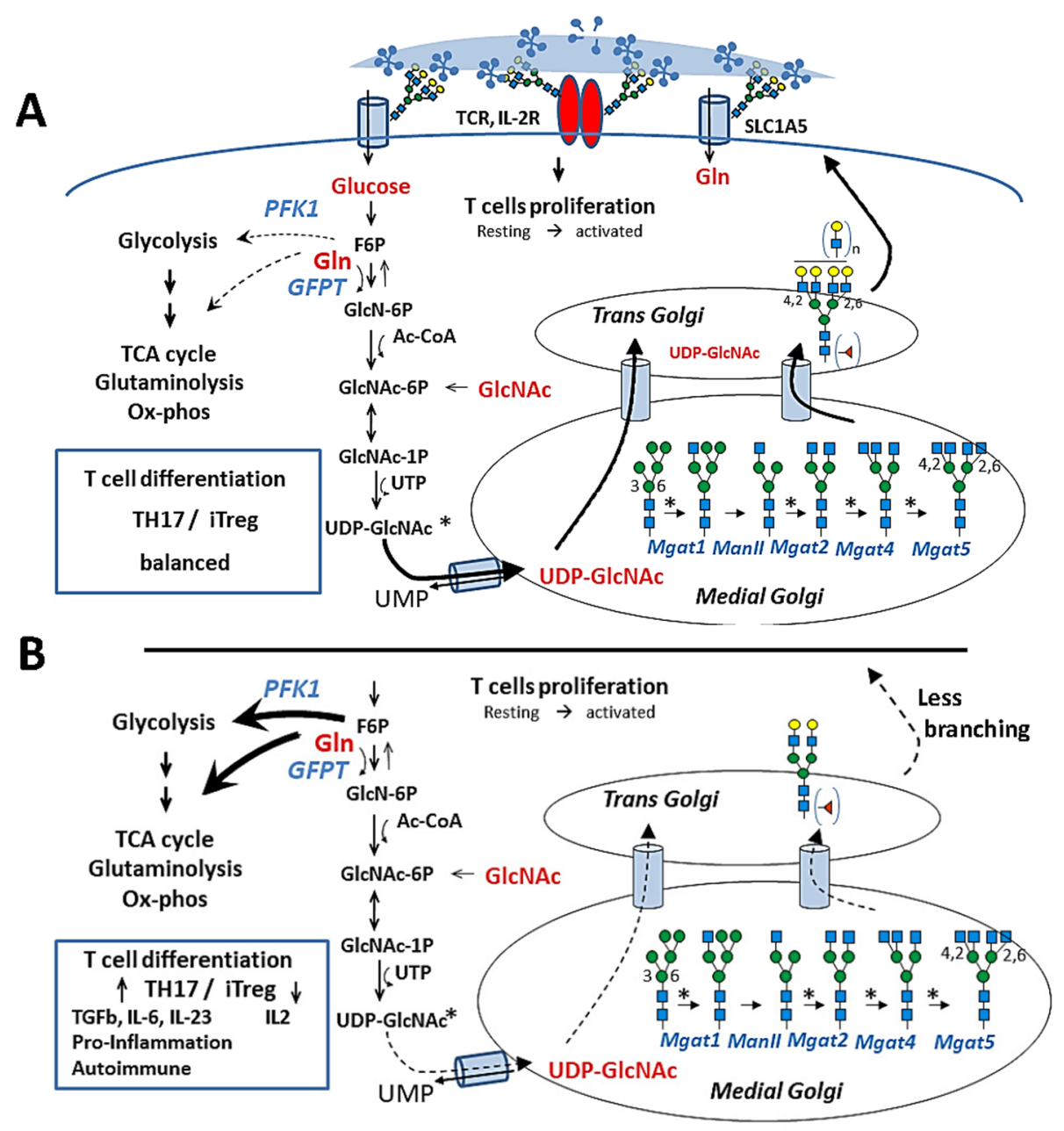

Fig. 5. Metabolic regulation of HBP and $N$-glycan branching. (A) Glucose is metabolized into fructose 6-phosphate, which supplies both glycolysis and the HBP. Ooxidative phosphorylation has modest needs for glucose, allowing the HBP to effectively compete with glycolysis for glucose flux to fructose-6-phosphate and thereby drive UDP-GlcNAc biosynthesis, $N$-glycan branching and balanced T cell responses. (B) Rapidly proliferating $\mathrm{T}$ cells switch from oxidative phosphorylation to aerobic glycolysis and glutaminolysis. This reduces flux of fructose-6-phosphate and glutamine into the HBP, thereby lowering UDP-GlcNAc biosynthesis and $N$-glycan branching to drive pro-inflammatory T cell responses. Thus, a primary function of aerobic glycolysis and glutaminolysis is to co-operatively limit metabolite supply to $N$-glycan biosynthesis, an activity with widespread implications for autoimmunity and cancer (52). Note Gal-3 as shown is only one of many possible microstates for self-association in the lattice.

pacity to cross-link $\beta$-galactoside-containing glycoconjugates. With the subsequent emergence of the galectin gene family in animals, $\beta$-galactoside binding activity has been conserved, and unencumbered by covalent linkage with other activities. Galectin CRDs have not been found in larger multi-domain proteins, as is common for other PTM-binding folds such as SH2 and PTB phosphotyrosine-binding domains (80). These observations are consistent with the adaptor function regulated in large part by $\beta$-galactoside ligand multiplicity and the modest affinities of galectin CRD domains which allow stochastic cross-linking.

However, some CRDs have evolved additional affinities for specific peptide sequences in cytosolic proteins with important adaptor functions. Cytosolic Gal-3 is reported to bind $\beta$-catenin, which could be inhibited by lactose $(81,82)$. Similarly, Gal-3 and
Gal-1 association with pre-mRNA splicing and nuclear ribonucleoprotein complexes are inhibited by lactose (82-84). This suggests that peptide binding occurs at the $\beta$-galactoside site in the CRD. The intracellular pathogen Salmonella Typhimurium cause vesicle damage, which disrupts lysosomal vesicles and exposes $N$-glycans to the cytosol which act as an "eat-me" signals that recruit galectins (85). While the N-terminal CRD of galectin-8 binds LacNAc, the C-terminal CRD also binds a nine amino acid sequence in NDP52, which in turn, recruits NAP1, TANK, ubiquitin receptors leading to autophagy and suppression of Salmonella $(86,87)$. Lysosomal membrane proteins (LAMP) are major carriers of $\mathrm{N}$-glycan chains with poly $N$-acetyllactosamine chains $(88,89)$. The Cterminal CRD of Gal-8 has evolved to recognize both the peptide and at lower affinity, $\beta$-galactosides. The bacteria-associated "eat- 
me" signals can be mediated by either Gal-8 or K48- K63-linked ubiquitin chains on the Salmonella; either suffices to recruit TBK1, which is essential for cell-autonomous immunity against infections.

\section{J. The Bigger Picture}

It is instructive to consider similarities with other multivalent PTM systems (see review (11)). As described above for the galectin lattice (20), the order of substrate activation by titration of PTM enzyme activity and site multiplicity has been described for protein phosphorylation by $\mathrm{Cdk} 1$ in the G1/S transition (90) and polyubiquitination by $\mathrm{APC} \backslash \mathrm{C}$ during exit from mitosis (91-93). The PTM modifiable sites often occur frequently in protein sequences, and sites function together acting as epitopes for binding partners (94, 95). Clustering of modifiable sites in the sequences of authentic protein substrates permits rapid and efficient processive modification. Specificity emerges in large part from the cooperative effects of PTM site clustering and chain formation, while decoy-substrates promote inhibitor ultrasensitivity, thereby tuning the stimulus- response relationships $(90,96)$. PTM dependent interactions with adaptor proteins are dynamic "jumping" between sites (i.e., stochastic binding-microsecond $\left.\mathrm{T}_{1 / 2}\right)(16,97)$, with cross-linking that can form lattice or liquid-droplet membrane-less organelles (8-10). Multivalent systems can buffer mutational loss or gain of PTM sites, thereby facilitating evolution of site positions (78, 79, 98). As such, the advantages are based on bioequivalence, multivalent interactions, evolvability of sites and tunable sensitivity of stimulation-response relationships by metabolism.

\section{Acknowledgments}

JWD and IRN's research is supported by grants from the Canadian Institutes of Health Research (MOP-126183, MOP136789, MOP-126029, NSERC), and Canada Research Chairs Program. M.D.'s research is supported by the National Institute of Allergy and Infectious Diseases (R01 AI108917) and the National Center for Complementary and Integrative Health (R01 AT007452).

\section{References}

1. Seetharaman, J., Kanigsberg, A., Slaaby, R., Leffler, H., Barondes, S. H., and Rini, J. M. (1998) J. Biol. Chem. 273 , 13047-13052.

2. Barondes, S. H., Cooper, D. N., Gitt, M. A., and Leffler, H. (1994) J. Biol. Chem. 269, 20807-20810.

3. Grigorian, A., and Demetriou, M. (2010) Methods Enzymol. 480, 245-266.

4. Nabi, I. R., Shankar, J., and Dennis, J. W. (2015) J. Cell Sci. 128, 2213-2219.

5. Cooper, D. N. (2002) Biochim. Biophys. Acta 1572, 209-231.

6. Herrmann, J., Turck, C. W., Atchison, R. E., Huflejt, M. E., Poulter, L., Gitt, M. A., Burlingame, A. L., Barondes, S. H., and Leffler, H. (1993) J. Biol. Chem. 268, 26704-26711.

7. Lin, Y. H., Qiu, D. C., Chang, W. H., Yeh, Y. Q., Jeng, U. S., Liu, F. T., and Huang, J. R. (2017) J. Biol. Chem. 292 , $17845-17856$.

8. Li, P., Banjade, S., Cheng, H. C., Kim, S., Chen, B., Guo, L., Llaguno, M., Hollingsworth, J. V., King, D. S., Banani, S. F., Russo, P. S., Jiang, Q. X., Nixon, B. T., and Rosen, M. K. (2012) Nature 483, 336-340.

9. Brangwynne, C. P., Eckmann, C. R., Courson, D. S., Rybarska, A., Hoege, C., Gharakhani, J., Julicher, F., and Hyman, A. A. (2009) Science 324, 1729-1732.

10. Kato, M., Han, T. W., Xie, S., Shi, K., Du, X., Wu, L. C., Mirzaei, H., Goldsmith, E. J., Longgood, J., Pei, J., Grishin, N. V., Frantz, D. E., Schneider, J. W., Chen, S., Li, L., Sawaya, M. R., Eisenberg, D., Tycko, R., and McKnight, S. L. (2012) Cell 149, $753-767$.

11. Dennis, J. W. (2015) Trends Biochem. Sci. 40, 673-686.

12. Zielinska, D. F., Gnad, F., Schropp, K., Wisniewski, J. R., and Mann, M. (2012) Mol. Cell 46, $542-548$.

13. Dennis, J. W., and Brewer, C. F. (2013) Mol. Cell. Proteomics 12, 913-920.

14. Hirabayashi, J., Hashidate, T., Arata, Y., Nishi, N., Nakamura, T., Hirashima, M., Urashima, T., Oka, T., Futai, M., Muller, W. E., Yagi, F., and Kasai, K. (2002) Biochim. Biophys. Acta 1572, 232-254.

15. Lee, R. T., and Lee, Y. C. (2000) Glycoconj. J. 17, 543-551.

16. Dam, T. K., and Brewer, C. F. (2008) Biochemistry 47, 8470-8476.

17. Stowell, S. R., Arthur, C. M., Mehta, P., Slanina, K. A., Blixt, O., Leffler, H., Smith, D. F., and Cummings, R. D. (2008) J. Biol. Chem. 283, 1010910123.

18. Do, K.-Y., Fregien, N., Pierce, M., and Cummings, R. D. (1994) J. Biol. Chem. 269, 23456-23464.

19. Losfeld, M. E., Scibona, E., Lin, C. W., Villiger, T. K., Gauss, R., Morbidelli, M., and Aebi, M. (2017) FASEB J. 31, $4623-4635$.

20. Lau, K. S., Partridge, E. A., Grigorian, A., Silvescu, C. I., Reinhold, V. N., Demetriou, M., and Dennis, J. W. (2007) Cell 129, $123-134$.

21. Sasai, K., Ikeda, Y., Fujii, T., Tsuda, T., and Taniguchi, N. (2002) Glycobiology 12, 119-127.

22. Demetriou, M., Granovsky, M., Quaggin, S., and Dennis, J. W. (2001) Nature 409, 733-739.

23. Dennis, J. W., Donaghue, T., Florian, M., and Kerbel, R. S. (1981) Nature 292, 242-245.

24. Kerbel, R. S., Lagarde, A. E., Dennis, J. W., and Donaghue, T. (1983) Mol. Cell. Biol. 2, $523-538$.

25. Cornil, I., Kerbel, R. S., and Dennis, J. W. (1990) J. Cell Biol. 111, 773-782.

26. Hebert, E., and Monsigny, M. (1994) Biol. Cell 81, 73-76.

27. Inohara, H., and Raz, A. (1994) Biochem. Biophys. Res. Commun. 201, 1366-1375.

28. Raz, A., and Lotan, R. (1981) Cancer Res. 41, 3642-3647. 
29. Yamashita, K., Ohkura, T., Tachibana, Y., Takasaki, S., and Kobata, A. (1984) J. Biol. Chem. 259, 10834-10840.

30. Yamashita, K., Tachibana, Y., Ohkura, T., and Kobata, A. (1985) J. Biol. Chem. 260, 3963-3969.

31. Pierce, M., and Arango, J. (1986) J. Biol. Chem. 261, 10772-10777.

32. Patnaik, S. K., Potvin, B., Carlsson, S., Sturm, D., Leffler, H., and Stanley, P. (2006) Glycobiology 16, 305-317.

33. Beheshti Zavareh, R., Sukhai, M. A., Hurren, R., Gronda, M., Wang, X., Simpson, C. D., Maclean, N., Zih, F., Ketela, T., Swallow, C. J., Moffat, J., Rose, D. R., Schachter, H., Schimmer, A. D., and Dennis, J. W. (2012) PLoS ONE 7, e43721.

34. Granovsky, M., Fata, J., Pawling, J., Muller, W. J., Khokha, R., and Dennis, J. W. (2000) Nat. Med. 6, 306-312.

35. Boscher, C., Zheng, Y. Z., Lakshminarayan, R., Johannes, L., Dennis, J. W., Foster, L. J., and Nabi, I. R. (2012) J. Biol. Chem. $287,32940-32952$.

36. Lagana, A., Goetz, J. G., Cheung, P., Raz, A., Dennis, J. W., and Nabi, I. R. (2006) Galectin binding to Mol. Cell Biol. $26,3181-3193$.

37. Goetz, J. G., Joshi, B., Lajoie, P., Strugnell, S. S., Scudamore, T., Kojic, L. D., and Nabi, I. R. (2008) J. Cell Biol. 180, $1261-1275$.

38. Partridge, E. A., Le Roy, C., Di Guglielmo, G. M., Pawling, J., Cheung, P., Granovsky, M., Nabi, I. R., Wrana, J. L., and Dennis, J. W. (2004) Science 306, 120-124.

39. Mkhikian, H., Grigorian, A., Li, C. F., Chen, H. L., Newton, B., Zhou, R. W., Beeton, C., Torossian, S., Tatarian, G. G., Lee, S. U., Lau, K., Walker, E., Siminovitch, K. A., Chandy, K. G., Yu, Z., Dennis, J. W., and Demetriou, M. (2011) Nat. Commun. $2,334$.

40. Johswich, A., Longuet, C., Pawling, J., Rahman, A. A., Ryczko, M., Drucker, D. J., and Dennis, J. W. (2014) J. Biol. Chem. $289,15927-15941$.

41. Stanley, P. (2016) J. Mol. Biol. 428, 3166-3182.

42. Cheung, P., and Dennis, J. W. (2007) Glycobiology 17, 767-773.

43. Lajoie, P., Partridge, E. A., Guay, G., Goetz, J. G., Pawling, J., Lagana, A., Joshi, B., Dennis, J. W., and Nabi, I. R. (2007) J. Cell Biol. 179, 341356.

44. Mendelsohn, R., Cheung, P., Berger, L., Partridge, E. A., Lau, K., Pawling, J., and Dennis, J. W. (2007) Cancer Res. 67, 9771-9780.

45. Schachter, H. (1986) Biochem. Cell Biol. 64, 163-181.

46. Hassinen, A., and Kellokumpu, S. (2014) J. Biol. Chem. 289, 26937-26948.

47. Climer, L. K., Dobretsov, M., and Lupashin, V. (2015) Front. Neurosci. 9, 405.

48. Thomson, M., and Gunawardena, J. (2009) Nature 460, 274-277.

49. Varki, A. (2011) Cold Spring Harb. Perspect. Biol. 3, a005462.

50. Valiant, L. (2013) Probably Approximately Correct: Nature's Algorithms for Learning and Prospering in a Complex World, Basic Books, Inc., New York, NY.

51. Lau, K. S., and Dennis, J. W. (2008) Glycobiology 18, 750-760.

52. Araujo, L., Khim, P., Mkhikian, H., Mortales, C. L., and Demetriou, M. (2017) eLife 6, 6.

53. Mkhikian, H., Mortales, C. L., Zhou, R. W., Khachikyan, K., Wu, G., Haslam, S. M., Kavarian, P., Dell, A., and Demetriou, M. (2016) eLife 5, 5.

54. Ohtsubo, K., Takamatsu, S., Gao, C., Korekane, H., Kurosawa, T. M., and Taniguchi, N. (2013) Biochem. Biophys. Res. Commun. $434,346-351$.

55. Ohtsubo, K., Takamatsu, S., Minowa, M. T., Yoshida, A., Takeuchi, M., and Marth, J. D. (2005) Cell 123, $1307-1321$.

56. Voss, M., Kunzel, U., Higel, F., Kuhn, P. H., Colombo, A., Fukumori, A., Haug-Kroper, M., Klier, B., Grammer, G., Seidl, A., Schroder, B., Obst, R., Steiner, H., Lichtenthaler, S. F., Haass, C., and Fluhrer, R. (2014) EMBO J. 33, 2890-2905.

57. Kuhn, P. H., Voss, M., Haug-Kroper, M., Schroder, B., Schepers, U., Brase, S., Haass, C., Lichtenthaler, S. F., and Fluhrer, R. (2015) Mol. Cell. Proteomics 14, 1584-1598.

58. Chen, I. J., Chen, H. L., and Demetriou, M. (2007) J. Biol. Chem. 282, 35361-35372.

59. Leppanen, A., Stowell, S., Blixt, O., and Cummings, R. D. (2005) J. Biol. Chem. 280, 5549-5562.

60. Halimi, H., Rigato, A., Byrne, D., Ferracci, G., Sebban-Kreuzer, C., ElAntak, L., and Guerlesquin, F. (2014) PLoS ONE 9, el11836.

61. Lepur, A., Salomonsson, E., Nilsson, U. J., and Leffler, H. (2012) J. Biol. Chem. 287, 21751-21756.

62. Ahmad, N., Gabius, H. J., Andre, S., Kaltner, H., Sabesan, S., Roy, R., Liu, B., Macaluso, F., and Brewer, C. F. (2003) J. Biol. Chem. 279, 1084110847.

63. Guttman, M., Weinkam, P., Sali, A., and Lee, K. K. (2013) Structure 21, 321-331.

64. Diehl, C., Genheden, S., Modig, K., Ryde, U., and Akke, M. (2009) J. Biomol. NMR 45, 157-169.

65. Diehl, C., Engstrom, O., Delaine, T., Hakansson, M., Genheden, S., Modig, K., Leffler, H., Ryde, U., Nilsson, U. J., and Akke, M. (2010) J. Am. Chem. Soc. 132, 14577-14589.

66. Boscher, C., and Nabi, I. R. (2013) Mol. Biol. Cell 24, 2134-2145.

67. Meng, F., Joshi, B., and Nabi, I. R. (2015) PLoS ONE 10, e0126056.

68. Zick, Y., Eisenstein, M., Goren, R. A., Hadari, Y. R., Levy, Y., and Ronen, D. (2002) Glycoconj. J. 19, 517-526.

69. Levy, Y., Arbel-Goren, R., Hadari, Y. R., Eshhar, S., Ronen, D., Elhanany, E., Geiger, B., and Zick, Y. (2001) J. Biol. Chem. $276,31285-31295$.

70. Nieminen, J., Kuno, A., Hirabayashi, J., and Sato, S. (2007) J. Biol. Chem. 282, 1374-1383.

71. Yang, E. H., Rode, J., Howlader, M. A., Eckermann, M., Santos, J. T., Hernandez Armada, D., Zheng, R., Zou, C., and Cairo, C. W. (2017) PLoS ONE 12, e0184378.

72. Ryczko, M. C., Pawling, J., Chen, R., Abdel Rahman, A. M., Yau, K., Copeland, J. K., Zhang, C., Surendra, A., Guttman, D. S., Figeys, D., and Dennis, J. W. (2016) Sci. Rep. 6, 23043.

73. Lee, S. U., Grigorian, A., Pawling, J., Chen, I. J., Gao, G., Mozaffar, T., McKerlie, C., and Demetriou, M. (2007) J. Biol. Chem. 282 , $33725-33734$.

74. Zhou, R. W., Mkhikian, H., Grigorian, A., Hong, A., Chen, D., Arakelyan, A., and Demetriou, M. (2014) Nat. Immunol. 15, $1038-1045$.

75. Li, C. F., Zhou, R. W., Mkhikian, H., Newton, B. L., Yu, Z., and Demetriou, M. (2013) J. Neuroimmunol. 256, 71-76.

76. Grigorian, A., Araujo, L., Naidu, N. N., Place, D., Choudhury, B., and Demetriou, M. (2011) J. Biol. Chem. 286, $40133-40141$.

77. Grigorian, A., Lee, S. U., Tian, W., Chen, I. J., Gao, G., Mendelsohn, R., Dennis, J. W., and Demetriou, M. (2007) J. Biol. Chem. 282, 2002720035.

78. Williams, R., Ma, X., Schott, R. K., Mohammad, N., Ho, C. Y., Li, C. F., Chang, B. S., Demetriou, M., and Dennis, J. W. (2014) PLoS ONE 9, 
e86088.

79. Dennis, J. W. (2017) Curr. Opin. Chem. Biol. 41, 1-11.

80. Jin, J., and Pawson, T. (2012) Philos. Trans. R. Soc. Lond. B Biol. Sci. 367, 2540-2555.

81. Shimura, T., Takenaka, Y., Tsutsumi, S., Hogan, V., Kikuchi, A., and Raz, A. (2004) Cancer Res. 64, 6363-6367.

82. Patterson, R. J., Haudek, K. C., Voss, P. G., and Wang, J. L. (2015) Methods Mol. Biol. 1207, 431-449.

83. Dagher, S. F., Wang, J. L., and Patterson, R. J. (1995) Proc. Natl. Acad. Sci. U.S.A. 92, 1213-1217.

84. Fritsch, K., Mernberger, M., Nist, A., Stiewe, T., Brehm, A., and Jacob, R. (2016) BMC Cancer 16, 502.

85. Thurston, T. L., Wandel, M. P., von Muhlinen, N., Foeglein, A., and Randow, F. (2012) Nature 482, 414-418.

86. Thurston, T. L., Boyle, K. B., Allen, M., Ravenhill, B. J., Karpiyevich, M., Bloor, S., Kaul, A., Noad, J., Foeglein, A., Matthews, S. A., Komander, D., Bycroft, M., and Randow, F. (2016) EMBO J. 35, 1779-1792.

87. Kim, B. W., Hong, S. B., Kim, J. H., Kwon, D. H., and Song, H. K. (2013) Nat. Commun. 4, 1613.

88. Heffernan, M., and Dennis, J. (1989) Cancer Res. 49, 6077-6084.

89. Carlsson, S. R., and Fukuda, M. (1990) J. Biol. Chem. 265, 20488-20495.

90. Kim, S. Y., and Ferrell, J. E. Jr. (2007) Cell 128, 1133-1145.

91. Rape, M., Reddy, S. K., and Kirschner, M. W. (2006) Cell 124, 89-103.

92. Lu, Y., Wang, W., and Kirschner, M. W. (2015) Science 348, 1248737.

93. Lu, Y., Lee, B. H., King, R. W., Finley, D., and Kirschner, M. W. (2015) Science 348, 1250834.

94. Nash, P., Tang, X., Orlicky, S., Chen, Q., Gertler, F. B., Mendenhall, M. D., Sicheri, F., Pawson, T., and Tyers, M. (2001) Nature 414, 514-521.

95. Csizmok, V., Orlicky, S., Cheng, J., Song, J., Bah, A., Delgoshaie, N., Lin, H., Mittag, T., Sicheri, F., Chan, H. S., Tyers, M., and Forman-Kay, J. D. (2017) Nat. Commun. 8, 13943.

96. Ferrell, J. E. Jr., and Ha, S. H. (2014) Trends Biochem. Sci. 39, 556-569.

97. Klein, P., Pawson, T., and Tyers, M. (2003) Curr. Biol. 13, 1669-1678.

98. Holt, L. J., Tuch, B. B., Villen, J., Johnson, A. D., Gygi, S. P., and Morgan, D. O. (2009) Science 325, 1682-1686.

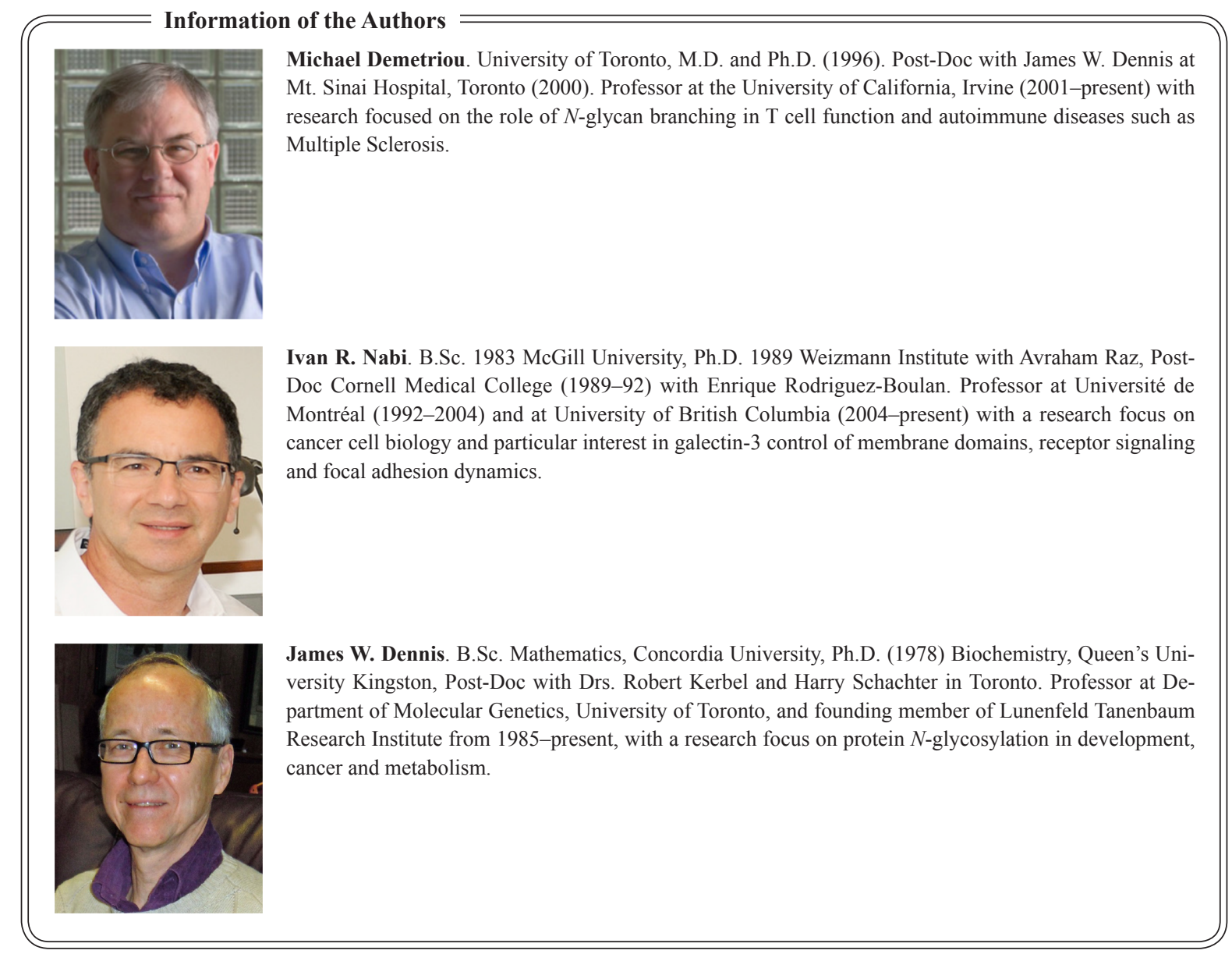

